\title{
THE RELATION OF ANATOMICAL FORM TO THE PRODUCTION OF FAVORABLE CONDITIONS IN CROWN AND BRIDGE-WORK
}

\author{
By H. E. FRIESELL, B.S., D.D.S., LL.D., F.A.C.D., and A. C. YOUNG, D.D.S., \\ Pittsburgh, Pennsylvania
}

(Read before the American Dental Association, Los Angeles, California, July 17-21, 1922)

$\mathrm{T}$ $\mathrm{HE}$ anatomical form of the teeth and jaws, while conforming to the same general characteristics, differs widely in individual cases. The jaws or their alveolar processes assume a more or less true semi-elliptical shape, opposing each other within the mouth cavity, the long axis passing through the center of the face.

The size and shape of these arches are of great importance, not only to the dental economy, but also as a factor in determining the general appearance of the face; any interference in their development having much bearing upon facial harmony and other normal lines of beauty. These arches carry in normal adult life, thirty-two teeth of varying size, shape and function, while the external surfaces of the arches are covered by the gum tissues, the gingiva extending between the teeth into the interproximal spaces. The health of these tissues is of vast importance as will be considered later.

Nature has so designed the teeth that each one will efficiently perform its individual function in the process of mastication of the food. The incisors are wedge shaped with the base of the wedge at the gingival margin from which the labial and lingual surfaces converge to form a straight cutting edge.

The cuspids, also wedge shaped, but somewhat inclined to be conical, so con- structed as to withstand stress in all directions, with long powerful roots, are admirably designed to perform their function of cutting, tearing and holding. The bicuspids and the molars with two and more cusps are designed to take care of the crushing, grinding process so necessary to the proper mastication of food.

The teeth, as the jaws, are arranged in the form of semi-ellipse, with the long axis between the centrals, the lowers on a slightly smaller curve than the uppers. The cutting edges of the incisors form a curved line from cuspid to cuspid, the line continuing with the buccal cusps of the bicuspids and molars to the third molar. The lingual cusps of the bicuspids and molars are within this line, the buccal cusps of the lowers interdigitating with the grooves between the cusps of the uppers. The line or plane in which the uppers and lowers occlude is not straight, but is curved so that in the lowers it presents a concavity, in the uppers is convex.

The teeth are all normally bioader at a point near the occlusal than at the neck. They have therefore, as placed in contact in the arch, V-shaped spaces between them, called interproximal spaces. In these spaces lie the gingival tissues. These spaces vary in width with varia. tions in shape and size of the teeth. The teeth being rounded on the proximal sur. 
- faces are in contact at but one point, the spaces therefore widening from this point toward buccal and lingual, to form embrasures. The position of this contact point, and its relation to the health of surrounding tissue is of the greatest importance, and especially from the standpoint of the present subject.

Our subject treats of the relation of anatomical form to the production of favorable conditions in crown and bridgework. In crown and bridge-work we place in the mouth various appliances, some reproducing the lost or broken down crown of a single tooth and depending for retention upon the remaining tooth structure; others, continuous masticating appliances replacing one or more lost teeth, and retained by various types of attachments upon remaining teeth.

For the purpose of our present discussion, we must consider crown and bridgework in two classes, one referring to single crowns, the other to bridges.

By "favorable conditions" we refer to those conditions which are essential to the continued comfort, usefulness ani health of the teeth and their supporting tissues. It is not within our field to describe the various types of crown and bridge-work and decide on their merits or demerits, but rather to draw attention to some conditions affected by their correct and incorrect form. We will consider the relationship of the form of crowns and bridges to the soft tissues and the possible effect upon these tissues. We will bear in mind that there is a possibility in incorrectly shaping the appliance of producing undue stress upon the abutment teeth, and upon the supporting alveolar process.

Let us first consider the conditions surrounding a tooth which needs restoration by means of a single crown. Anterior or posterior, we have to deal with a broken down tooth, which has lost its original contour or form. Very often in anterior cases, the entire crown of the tooth is broken off. Generally the contact with the adjacent teeth has been lost, due to the loss of tooth structure. This will allow a movement or crowding of the adjacent teeth from mesial and distal, with a loss of the space originally occupied by the tooth to be crowned.

We all recognize that these conditions, if not corrected, will eventually result in that chain of pathological changes running through gingivitis to pyorrhea and loss of the teeth. In addition to these conditions, there may be a change by elongation of the position of the occluding tooth or teeth.

We must bear all these things in mind while constructing a crown to restore the function of the broken down tooth. First the occlusal surface of the opposing tooth or teeth must be corrected so as to be in the proper plane of occlusion. If this is not done one or two teeth will bear unusual stress in the lateral movements of mastication. If the adjoining teeth have crowded together so as to partially close the space of the broken down tooth, they should be forced into correct position, and held there until the crown is placed.

Regardless of type, the crown should be so adapted at the gingival margin as to avoid all irritation to the gingiva. Of course, actual impingement of the crown upon the periodontal membrane will cause irritation, and is never permissible. In addition, the form or contrur of the crown must be arranged to protect the soft tissues. The normal tooth is shaped so as to do this, there being more or less flare or bell crown shape from the neck of the tooth toward the height of contour. In making a crown, this flare should be imitated and even accentuated in an effort to protect the soft tissues of the neck of the tooth from a direct impact of the particles of food.

On the proximal surfaces, proper contact with adjoining teeth is essential. The same rules govern the shaping of the proximal surfaces in crown and bridge- 
work as those given to the profession in Dr. Black's works on operative dentistry.

He says (Page 295, Vol. 2): "The usefulness of a filling depends directly upon the form given the proximal surface. There is perhaps more failure of fillings attributable to imperfect work in this, than in any other one thing." What is true of fillings, is true of crowns. If the contact point is not properly restored, particles or shreds of food will lodge in the interproximal space and, crowding the gum away, form a pocket for lodgment and decomposition. This will result in caries of the adjacent tooth or teeth, and disease of the gum tissue and periodontal membrane. Attention should be given the form of the contact point and the position it takes in relation to the other surfaces and to the adjacent tooth.

The form of contact between two teeth should be similar to the contact of two spheres or marbles, there being but one point of immediate contact. From this point the surfaces should be so formed as to slope toward occlusal, gingival, and into embrasure, that will allow the food taking its normal course through these embrasures to buccal and lingual.

Upon the buccal, labial and lingual surfaces, height of contour must be established, so the sweep of food is directed as to make impaction impossible. Instead, the food in masticating must be so directed as to strike at a tangent, scouring the surfaces of the soft tissues. These tissues are thus stimulated, rather than irritated by impact.

The form of the incisal and occlusal surfaces has its relative importance. This surface must be so shaped as to articulate properly with the opposing teeth, in normal occlusal plane. In posteriors, cusps should be established in proper position, with corresponding sulci and grooves. The necessity of a properly-shaped occlusal surface so that the tooth can take its place with its fellows in breaking up the bolus of food is understood and should be observed by all who construct these appliances. The relation of the occlusal to the axial surfaces should be so arranged that the cheek and tongue are protected from injury in mastication. The marginal ridges and the cusps if properly placed will not pinch these soft tissues as the buccal and lingual height of contour should hold them away.

We have some different conditions to deal with, if teeth are missing, and a bridge is decided upon as the correct method of restoration.

The abutment teeth have at least one proximal surface which is adjacent to the space left by extraction of a tooth. At times we must use as a supporting tooth one with teeth extracted mesially and distally. This tooth must be especially protected, both against irritation to the surrounding gum tissue and against undue strain to its weakened bony support. Between the abutments, we have to deal with a span of the ridge from which alveolar process has absorbed. Where the teeth have been lost for any length of time, the antagonizing teeth in the opposite jaw are sure to have elongated to a greater or less extent.

To construct a bridge to restore the lost teeth, and to make it anatomical in form, should be and is recognized as an impossibility. To a limited extent only, regardless of the type of bridge, are we able to completely restore the shape of the lost teeth. The shape given the socalled dummies, or pontics, should therefore be called hygienic rather than anatomic.

Where we have elongation of opposing teeth, the normal occlusal plane must be restored, before proceeding with the actual bridge construction. It is impossible to build up the lost tooth contour if a great part of the gingivo-occlusal space is taken up by elongated opposing teeth, neither is it possible to construct a serviceable bridge, as the triturating motion, so necessary to proper function of the masticating organ, is lost or interferred with. With only one movement possible, opening and closing, the food 
can be crushed, not ground. If a lateral motion is attempted, the only contact is on the cusps of the elongated tooth. In constructing the abutments for a bridge, the same conditions apply as when making a single crown, except that at least one proximal surface will be attached to the body of the bridge. At this point, the form of the work needs especial care. The gingiva is not normal here, and any irritation or impaction will lead readily to the formation of a pocket. The proximal surface of the abutment should be slightly overcontoured, and the dummy should be attached so that, to every extent possible the food can sweep through the embrasures. A solid contact soldered from buccal to lingual is sure to allow for collection of debris and more or less gingival irritation.

The body of the bridge, as mentioned before, cannot in many cases be constructed to anatomical form, but can only be made to approach tooth form to the extent that the nature of the appliance will allow.

The occlusal surfaces should articulate properly with the opposing teeth, and have such a form as will allow the food to be broken up in mastication, rather than caught on the bridge surface and crushed. The latter condition, in addition to being inefficient, throws undue strain on the abutment teeth and alveolus, and will soon result in pathological changes. Gateways must be formed between the dummies to imitaie the embrasures between natural teeth. These will allow of proper mastication, and due to the sweeping action of the foods stimulate and clean the tissues of the ridge beneath the bridge.

'The surfaces of the bridge adjacent to the tissues must be cleanly, and proper contour will tend to keep them so. These surfaces must be convex, so that the sweep of the food, and the motions of the tongue, lips and cheeks will have a cleansing action upon them. Concave surfaces not only resist this natural cleansing, but resist mechanical scouring.

Alignment with the other teeth in the semi-ellipse of the arch is another necessity. If the bridge be out of alignment it is impossible to adapt it to the various positions in mastication. Certain thrusts of the jaw are sure to bring a strain upon the supporting structures, that will soon destroy them and cause loss of the appliance.

As with crowns, the forms presented to the cheeks and tongue should be so arranged that these tissues are held a little apart from the actual points of occlusal contact, to protect them from being caught and crushed between the bridge and the opposing teeth.

It will be seen from a consideration of these facts that anatomical form has the most important relationship to the successful crown and bridge result, and that a cleanly, efficient and lasting piece of work can only be constructed by keeping in mind the anatomy of the teeth, and surrounding tissues.

Due to the nature of the work, we can only imitate this form, but the ideal must be kept constantly in mind. 